\title{
Research on Computer network multimedia transmission system
}

\author{
Chunxi Zhao \\ Jilin Agricultural University, Changchun 130118,China \\ 10821395@qq.com
}

\begin{abstract}
Keywords: Streaming media RTP protocol, RTCP protocol
\end{abstract}
\begin{abstract}
With the development of computer network, there are more and more multimedia real-time transmission methods. The traditional stand-alone multimedia synchronization method is unable to solve out of the sync problems due to the unstable factors in the network transmission. Therefore synchronization method for streaming media communication research is very important. RTP and RTCP protocol is geared to the needs of multimedia network transmission and development, on the application of RTP and RTCP protocol has many places worthy to be discussed. Based on the multimedia real-time network of RTP and RTCP protocol, the implementation of the specific researches are studied, and in a local area network environment the tested conclusions are given.
\end{abstract}

\section{Introduction}

Multimedia technology can make computer integrated processing sound, image and video, and let the way people using computers become diversification. As to the development of broadband network, people hope to be able to enjoy continuous online multimedia programs. As a cross subject of multimedia and network streaming media technology emerged. Streaming media refers to the network used in the continuous time base media streaming technology, streaming media technology is used to publish on the Internet and download the technology of multimedia data streams. The main characteristics of streaming media are to download and watch, in the form of "water" to finish the transmission of digital media.

\section{The streaming media synchronization method}

The traditional streaming media synchronization method for stand-alone multimedia synchronization method basically has the following three Steps: (1) notation. The sender terminal sends a synchronous flag, the receiver receives synchronous tags, used to synchronous processing various media to achieve the goal of both sides communication synchronization. This method is simple, the cost is not big. The method is able to ensure real-time, don't need synchronous clock with good compatibility. The defect is this method changed data structure, not directly used for the connection and application, does not support complex synchronization, multimedia stream doesn't suit for different sources.(2) the multiplex synchronous method. The method to reuse multiple media stream data into a data stream or a packet, which makes them naturally in a media conference in multimedia transmission system, build a multimedia virtual circuit for each session (MVC). The sender use all media streams multiplexing on the MVC, compound into a sequential organization of packet flow to ensure the synchronization between the media. To the receiver there are a variety of media flow solutions for reuse from the MVC, submitted to the user process. The advantages of this method is to realize a simple, less overhead, transport guarantee real-time, apply to access data; At the same time there are also disadvantages. The flexibility is poor, unable to meet the requirements of different levels of synchronization. It can't lose relative unneeded messages. It's unable to process different source of media flow. This process will change the data structure, and the connection is indirect. (3) the timestamp synchronization method. The method is using by putting the data flow between each media unit to join united timestamp (time code). With the same timestamp media unit are able to play at the same time, in order to achieve the purpose of synchronization between media. This method need not change the data flow, no additional synchronization channel, which can be 
widely used. The downside is that the choice of the relative time scale and the determination of the timestamp operation are relatively complex, need certain overhead for synchronization, lost is out of sync.

\section{The multimedia real-time transmission based on RTP and RTCP protocol research and implementation}

RTP is a kind of real-time transport protocol provides end-to-end transport services, to support the single objective and multiple objectives for the transmission of real-time data network services. Using RTP protocol application running on RTP, and the implementation of RTP, programs run on the upper deck of the UDP, the purpose is to use UDP port number. As shown in figure 1, RTP, may be regarded as a child of the transport layer. Audio and video data generated by the multimedia application block is encapsulated in the RTP packets, each of the TRP packets is encapsulated in the UDP messages section, and then encapsulated in PI data packets.

\begin{tabular}{|c|}
\hline Application \\
\hline RTP \\
\hline UDP \\
\hline IP \\
\hline Data link \\
\hline Physical \\
\hline
\end{tabular}

Figure 1 RTP protocal of transmission layer

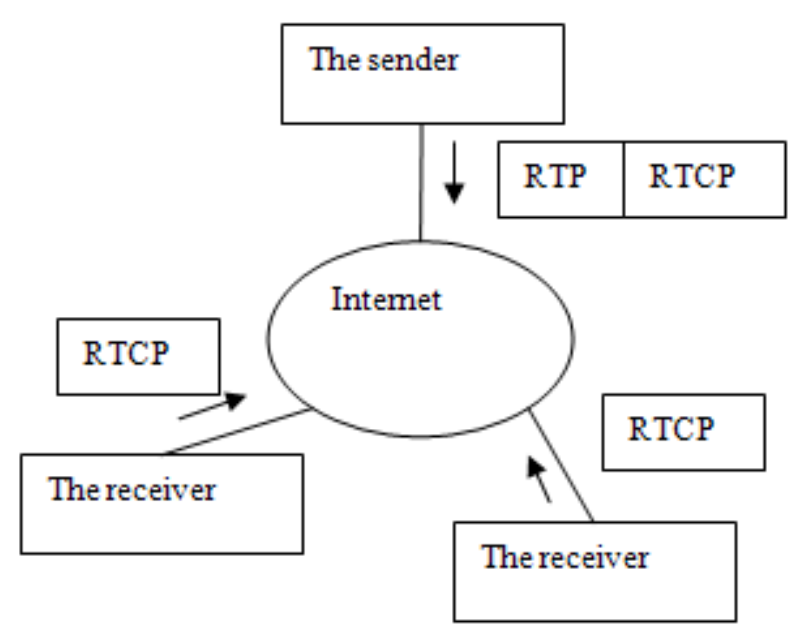

Figure 2 RTCP control packet

RTP header by 12 bytes of header information and payload domains, including four is a packet header field, including: payload types (Payload type), worthy of the serial number (sequence number), $\mathrm{J}$ time slaughter (timestamp) and source identifier (synchronization source identifier), etc. The application of multimedia network the RTP and RTCP are used together, especially in the multi-objective more attractive in the radio. When one or more from the sender to the receiver of multiple radio voice or video, in the RTP session, each participant periodically send RTCP control packet to all other participants, as shown in figure 2.

The function of RTCP described as follows: (1) The QoS control and congestion control RTCP packet contains the necessary information for QoS control. Recently sent by the application of video data and audio data send to produce a report, which mainly contains the synchronization between the media information, and to send the packet and the cumulative number of bytes. The send report allows the receiver to estimate the actual data rate. Receives the data of the receiver must produce a receiving report, the report contains the packet sequence number, the number of packet loss, delay jitter, and time.(2) Media synchronization between send RTCP report contains a real-time display and corresponding RPT timestamp, to control the synchronization between different media. Take the synchronization of video and audio for example. (3) The size of the traffic estimation and measure each communicating members periodically send RTCP packet, need both the latest control information, at the same time, must be control information transmission volume ratio in the data traffic will be limited to a small range, reduce the consumption of network bandwidth, generally limited to around 5\%. 


\section{Implementation of the multimedia real-time transmission}

The current network bandwidth requirements of multimedia data compression can be suitable for network transmission, when implemented, we adopt the MPEG compression standard IV, realized the high compression ratio. Use caching system to compensate for the influence of delay and jitter, and in the cache implementation of packet reordering, guarantee the order of the packets correctly, so that the media data can be output in a row, and won't because the network temporarily blocked that play a pause. The realization of video transmission is using RTP/RTCP as the transport protocol to transmit real-time video data Server to SoQ control system. The server system block diagram is as shown in figure 3.

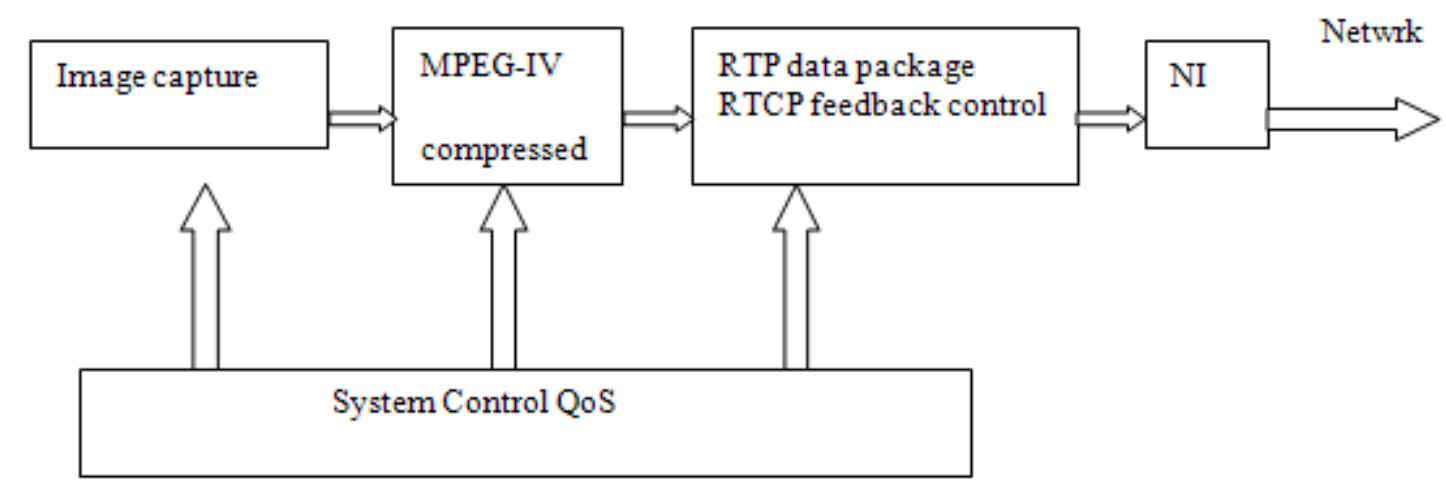

Figure 3 Server system block diagram

Multimedia real-time transmission data process and control process With Sending the encoder output compressed a frame of video data, submitted to RTP packet processing module, we will first create a RTP, thread, a video data in accordance with the requirements are decomposed into a series of small packets for network transmission, in the order send serial number of tag data packets, and according to the current sampling clock on time stamp, tag format of video data compression, and parameters such as packet header of this agreement, to the transmission module sent to complete. Transmission module according to the received RTCP packets of feedback information, improve the sending the sending rate, even the encoding format.

Media transmission rate feedback control when in the implementing of end-to-end transmission of multimedia, the client will periodically send RTCP control information to the server message. The receiver report RR mainly provides data transmission to receive quality feedback, including the maximum number of packets received, the number of lost packets, calculate the round-trip delay on sending and receiving. In practice, the widely used control server sends the packet loss rate. Assuming that the maximum number of packets received for $B_{\max }$, number of packets lost are $B_{\text {lost. }}$. The actual packet loss $L$ at the receiving end will be

$$
\mathrm{L}=\mathrm{B}_{\text {lost }} / \mathrm{B}_{\max }
$$

In the end-to-end transmission, you can set the upper limit of packet loss as $\mathrm{L}_{\text {min }}$ and lower limit $\mathrm{L}_{\max }$

When $\mathrm{L}>\mathrm{L}_{\max }$, reduce the transmission speed;

When $\mathrm{L}_{\min }<\mathrm{L}<\mathrm{L}_{\max }$, keep sending rate;

When $\mathrm{L}<\mathrm{L}_{\text {min. }}$, increase the transmission speed. 
The key lies in how to select the upper limit of packet loss $\mathrm{L}_{\max }$ and lower limit of packet loss $\mathrm{L}_{\min }$. The data of $\mathrm{L}_{\max }$ presents high data transmission, but also can accept. The selection of $\mathrm{L}_{\min }$ need to make balance of the area is enough big, avoid oscillation.

Intelligent flow technology Multimedia multicast network, each client connection speed will be a very big difference. If you are using a single transmission rate, for the client connection rate is less than the transmission rate, buffer phenomenon will happen frequently. For the client connection speed is greater than the transfer rate, receiving the effect is not ideal. It is hard to make full use of network resources. In order to realize the different network connection rate of the client to provide different transmission rate, according to the feedback of the multimedia transmission rate to control the transmission rate.(1)Use RTPSessionSetBandwidth () to set network bandwidth, according to a certain bandwidth, provide the corresponding transmission rate. (2) The server automatically detect bandwidth changes, using RTPSessionGetBandwidth () to obtain the current network bandwidth, change according to the bandwidth compression of image quality, provide different transmission rate.(3)When the connection rate is low, the network under the condition of poor conditions, guarantee the key frames is preferred, appropriate discard some non-critical data frames.

\section{Results}

Experiment respectively by using narrow-band transmission line and 10M LAN transmission, the video is used after camera field acquisition, by compression module compression become a worker format MPEG video streams, pixel at 352 x288.In the experiment, after a few seconds of delay, the client can accept the data. On the narrow-band transmission line, can reach 12 frames per second, at $10 \mathrm{~m}$ LAN transmission can achieve continuous stream of 25 frames per second. Experiments show that using RTP/RTCP protocol we implemented end-to-end multimedia network transmission, real-time transmission obtained satisfactory effect.

\section{Conclusions}

The proposed approach is streaming video and audio timestamp establish a mapping relationship, but this method is easier to understand and implement. In this paper, design of which has the function of network real-time streaming multimedia processing and transmission system, can be widely used in remote monitoring, meeting real-time requirements such as TV and video phone is higher. However, to really on the Internet and other grouping network multimedia conference businesses, also need to consider issues of privacy and security.

\section{References}

[1] Douglas E Come r, David L Stevens. Connection between networks by using TCP / IP( 3rd volume client- server program and application Linux /POSIX socket edition) [M ] . Translated by ZHAO Gang, LIN Yao, JIANG Hui et al Beijing: Electron Industry Publishing House, 2001.

[2] ZHAO Jin. The software design and realization of streaming media transportation system based on RTP protocol family[ D] .Master degree paper of SCUT, 2004.

[3] ZHANG Li-yan. The research of RSVP realization and its real-time application[ D]. M aster degree paper of SCUT, 1999.

[4] CU I Li WANG M in, JI Yi Study of synchronization of streaming media [ J]. Research of Computer application, 2005( 1): 73-75.

[5]REN Yan-zhen, YU Zhan-wu, HU Rui min. Controlling strategy of real-time da ta transportation and synchronization based on RTP /RTCP protocol [ J]. Computer Engineering and Application, 2003( 10): 144-147. 